\title{
PLEKHS1 Over-Expression is Associated with Metastases and Poor Outcomes in Papillary Thyroid Carcinoma
}

\author{
Xiangling Xing ${ }^{1}$, Ninni Mu ${ }^{2,3}{ }^{(}$, Xiaotian Yuan ${ }^{1, *(\mathbb{C},}$, Na Wang ${ }^{2}$, C. Christofer Juhlin ${ }^{2,3} \mathbb{C}^{\text {, }}$ \\ Klas Strååt ${ }^{1}$, Catharina Larsson ${ }^{2,3, *}$ and Dawei $\mathrm{Xu}{ }^{1}$ (D) \\ 1 Department of Medicine, Division of Hematology, Bioclinicum and Center for Molecular Medicine, \\ Karolinska University Hospital Solna and Karolinska Institutet, SE-171 76 Stockholm, Sweden; \\ Xiangling.Xing@ki.se (X.X.); Klas.Straat@ki.se (K.S.); Dawei.Xu@ki.se (D.X.) \\ 2 Department of Oncology-Pathology, Karolinska Institutet, Karolinska University Hospital Solna, Bioclinicum, \\ SE-171 76 Stockholm, Sweden; Ninni.Mu@ki.se (N.M.); na.wang@ki.se (N.W.); christofer.juhlin@ki.se (C.C.J.) \\ 3 Department of Clinical Pathology and Cytology, Karolinska University Hospital, \\ SE-171 76 Stockholm, Sweden \\ * Correspondence: Xiaotian.Yan@ki.se (X.Y.); Catharina.Larsson@ki.se (C.L.)
}

Received: 6 July 2020; Accepted: 29 July 2020; Published: 31 July 2020

\begin{abstract}
Pleckstrin homology domain containing S1 (PLEKHS1) is a poorly characterized factor, although its promoter mutations were identified in human malignancies including thyroid carcinoma (TC). This study was designed to determine PLEKHS1 promoter hotspot mutations in papillary and anaplastic thyroid carcinomas (PTCs and ATCs) and to evaluate if PLEKHS1 expression influences clinical outcome. The PLEKHS1 promoter mutation was observed in 1/93 of PTCs and none of 18 ATCs in our cohort; however, PLEKHS1 expression was aberrantly up-regulated in TCs compared to adjacent non-tumorous thyroid tissues. ATC tumors, an undifferentiated TC, exhibited the highest PLEKHS1 expression. In both TCGA and present cohorts of PTCs, PLEKHS1 gene methylation density was inversely correlated with its mRNA expression and demethylation at the PLEKHS1 locus occurred at two CpGs. Higher PLEKHS1 expression was associated with lymph node and distant metastases, and shorter overall and disease-free survival in our cohort of PTC patients. Importantly, PLEKHS1 over-expression predicted shorter patient survival in PTCs lacking TERT promoter mutations. Cellular experiments showed that PLEKHS1 over-expression enhanced AKT phosphorylation and invasiveness. Collectively, the PLEKHS1 gene demethylation causes its over-expression in PTCs. PLEKHS1 promotes aggressive behavior of TCs possibly by increasing AKT activity, and its over-expression predicts poor patient outcomes.
\end{abstract}

Keywords: metastasis; PLEKHS1; prognostic factors; promoter mutations; TERT; thyroid carcinoma

\section{Introduction}

Papillary thyroid carcinoma (PTC), the most common type of thyroid cancer (TC) originating from follicular thyroid cells, accounts for more than $80 \%$ of all thyroid malignancies [1,2]. The vast majority of PTCs exhibit an indolent nature with favorable outcomes, but $10-15 \%$ of localized and R0 resected PTCs will eventually relapse or metastasize, and develop treatment resistance, thereby leading to disease-related morbidity/mortality [1-4]. Thus, it is clinically important to precisely stratify these aggressive PTCs for timely intervention and close surveillance. A number of clinical variables including age, tumor size, extrathyroidal extension, lymph node status, and the occurrence of distant metastasis are routinely applied to correctly stage TCs in general. However, despite their usefulness in choosing therapeutic approaches, these factors remain insufficient to predict PTC progression and 
recurrence post-surgical treatment. Anaplastic thyroid carcinoma (ATC) is an undifferentiated form of TC that might originate from pre-existing well-differentiated thyroid carcinomas including PTC or occur de novo [1,2].

Recent advances in high-throughput next-generation sequencing technologies have led to a revolution in cancer genomics including PTCs and ATCs, providing deep insights into the molecular pathogenesis and facilitating the identification of new biomarkers for the disease prognostication [2,3,5-7]. Recurrent promoter mutations (C228T/C250T) of the telomerase reverse transcriptase (TERT) gene were recently identified in PTCs and ATCs, and the presence of this genetic event predicts poor patient outcomes [8-14]. Moreover, the TERT promoter mutation activates TERT gene transcription $[8,15]$, and higher TERT expression is similarly associated with aggressive PTCs $[9,11,14,16]$.

Although TERT promoter mutations are very helpful for PTC prognostication, they occur only in a small fraction (5-25\%) of patients $[8,14,16]$, and it remains challenging to accurately foresee the outcome of patients carrying a wild-type (wt) TERT promoter. In search for new prognostic factors, the Pleckstrin homology domain containing S1 (PLEKHS1) gene has attracted our attention, because hotspot PLEKHS1 promoter mutations (Figure 1A) have also been shown to occur in different types of human cancer including TC and other malignancies where TERT promoter mutations are frequently observed $[17,18]$. In the present study, we analyzed the PLEKHS1 gene for its promoter mutation and expression. Our results show that the PLEKHS1 hotspot promoter mutations are rare, but its higher expression is significantly associated with metastasis and shorter patient survival in PTCs.

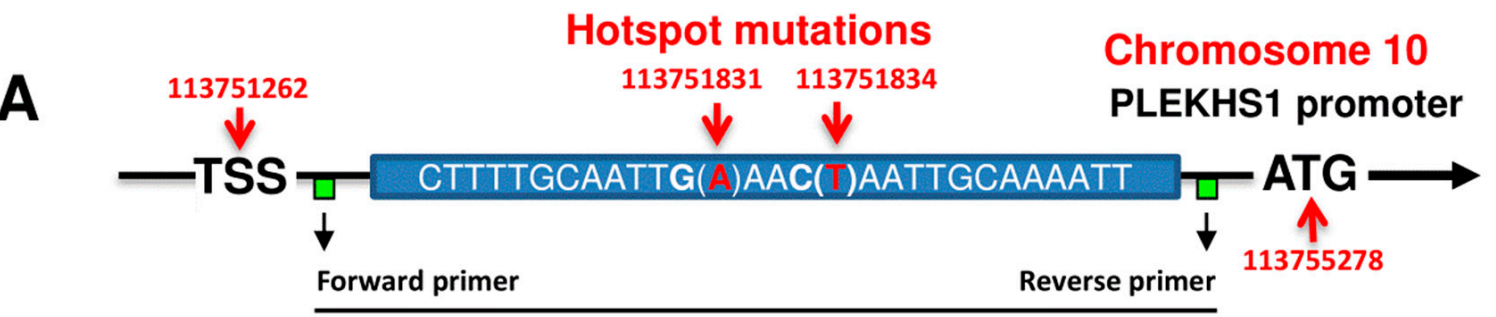

241 bp fragment for Sanger sequencing

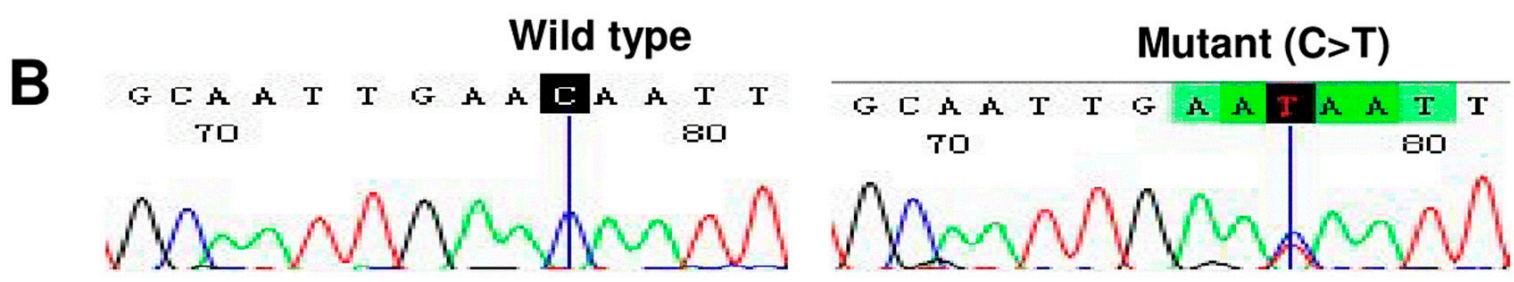

Figure 1. PLEKHS1 promoter mutations are rare in PTCs. (A) Schematics of hotspot mutations in the PLEKHS1 promoter (based on the GRCh38.p13). Two mutation hotspots on chromosome 10 are indicated (G > A 113751831) and (C > T 113751834), respectively. Of note, these two mutations are flanked by stretches of $10 \mathrm{bp}$ on both sides that are palindromic to each other. TSS: Transcription start site. The locations of primers for Sanger sequencing are indicated. (B) Wild-type (wt) and mutant PLEKHS1 promoter sequences as determined using Sanger sequencing in PTC tumors. A representative chromatogram of a PTC tumor with a wt PLEKHS1 promoter is shown in the left panel. Shown in the right panel is the only PTC tumor carrying the PLEKHS1 promoter mutation.

\section{Results}

\subsection{The Rarity of PLEKHS1 Hotspot Promoter Mutations in PTCs and ATCs}

A hotspot PLEKHS1 promoter mutation was shown to occur in one PTC out of nine analyzed TCs, in an earlier report [17] (Figure 1A), which suggests that this genetic event might be recurrent 
in PTC. We thus analyzed the PLEKHS1 promoter region for the hotspot mutations in five TC cell lines, primary PTC tumors from 93 patients and ATCs from 18 patients using Sanger sequencing. The sequencing analyses revealed that all five cell lines were wild-type, while only one PTC tumor was mutation-positive (Figure 1B). This was derived from a 75 years old female patient that in addition to the PLEKHS1 promoter-hotspot mutation also carried a TERT promoter C250T mutation but lacked the $B R A F^{\mathrm{V} 600 \mathrm{E}}$ mutation. She had lymph node and distant metastases, and relapsed or progressed in her disease 6 months after surgery. These results clearly demonstrate the rarity of the PLEKHS1 promoter mutation in PTCs and ATCs.

\subsection{PLEKHS1 Expression in TC Cell Lines and Primary PTC and ATC Tumors}

We then determined PLEKHS1 expression in five cell lines and 111 primary tumors (93 PTCs and 18 ATCs). PLEKHS1 mRNA was detected in all three ATC-derived cell lines with the highest expression in SW1736 cells. Interestingly, PTC-derived MDA-T32 and MDA-T41 cells expressed the lowest levels of PLEKHS1 mRNA (Figure 2A), and similar findings were obtained for PLEKHS1 protein levels in these cells (Figure 2A), indicating transcriptional control of this gene. We then analyzed PLEKHS1 expression in primary tumors and non-cancerous thyroid tissues. In the TCGA dataset, comparison between PTCs and non-cancerous thyroid tissue samples showed a significant increase in PLEKHS1 mRNA expression in tumors (239.8 (0-3445) vs. 624.9 (0-263865) median (Min-Max), $p<0.0001$ ) (Figure 2B). For PTCs and ATCs, their PLEKHS1 mRNA quantifications were $1.5(0.2-811.9)$ and 3.7 (1.0-85.6), (median (Min-Max)), respectively, in our cohort $(p<0.0001$, Figure 2C). Thus, ATC tumors expressed significantly higher levels of PLEKHS1 mRNA than did PTCs. In addition, 20 of 93 PTC tumors were also analyzed for their PLEKHS1 protein expression using immunoblotting assays. Their mRNA and protein levels of PLEKHS1 were in general consistent with each other, although variations occurred to certain extents and correlation was not statistically significant (Figure 2D, $r=0.338, p=0.145$ ). However, the analysis of combining both 5 TC cell lines and 20 primary PTC tumors revealed a significantly positive correlation between PLEKHS1 mRNA and protein (Figure 2E, $r=0.493, p=0.0123$ ). Taken together, these results obtained from cell lines and primary tumors suggest that PLEKHS1 mRNA over-expression aberrantly occurs in PTCs and ATCs. 
A
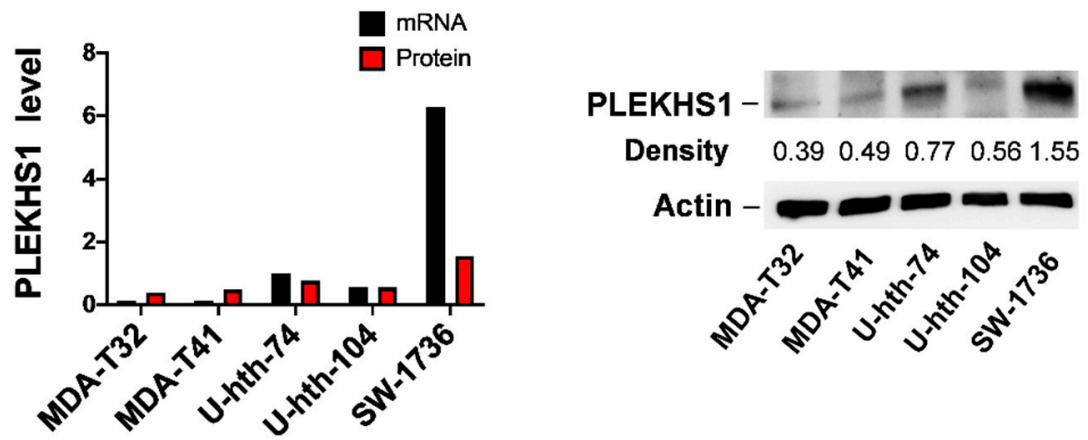

B
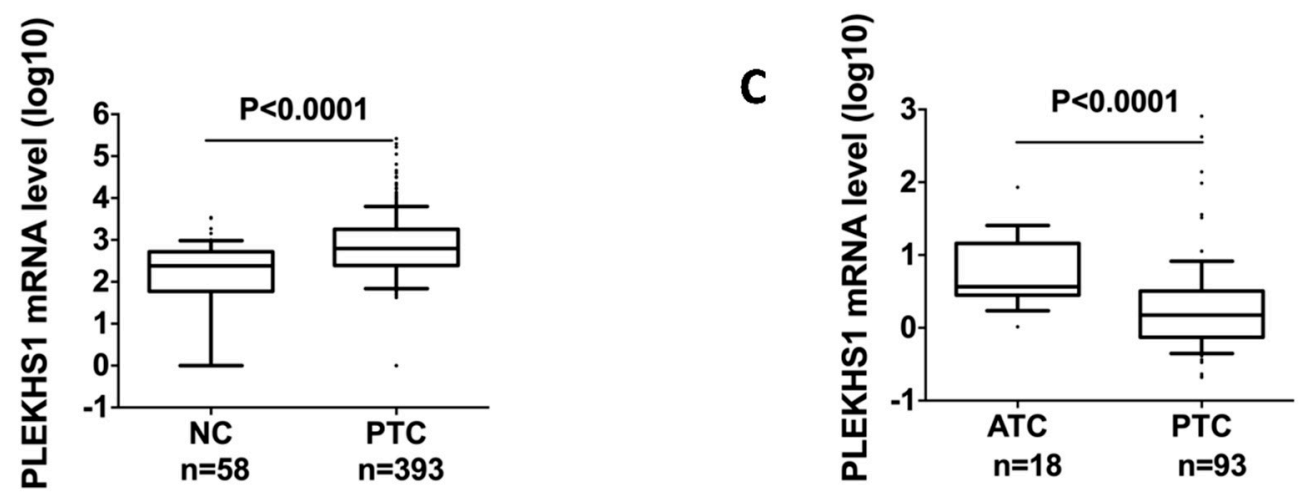

D

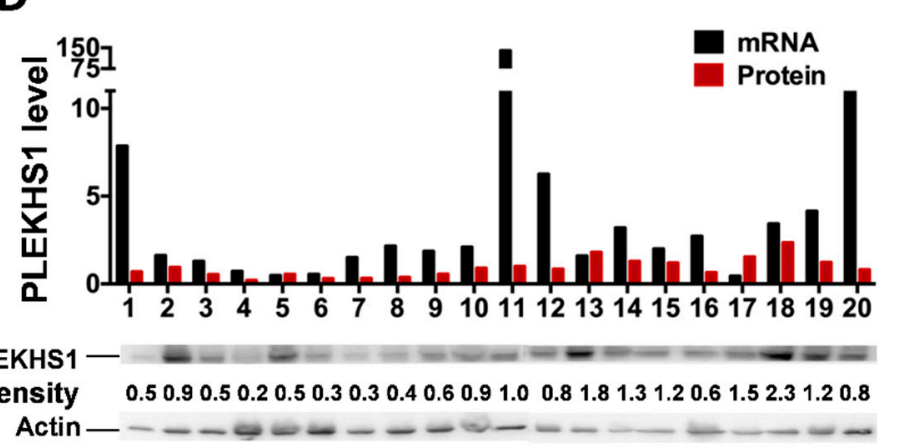

E

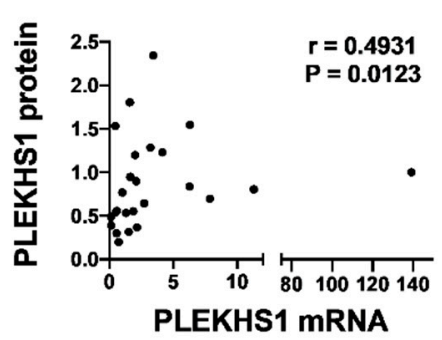

Figure 2. PLEKHS1 is over-expressed in thyroid cancer (TC)-derived cell lines and primary tumors. (A) PLEKHS1 expression in TC cell lines. Left and right: PLEKHS1 mRNA and protein expression levels as determined using qPCR and immunoblotting, respectively. The quantification results (band intensity) in the right panel are shown in the left panel. (B) Differential expression of PLEKHS1 mRNA between non-cancerous adjacent thyroid tissues and primary PTC tumors. The TCGA cohort of PTC tumors and adjacent tissues are analyzed and mRNA levels were expressed as FPKM (Fragments Per Kilobase Million). (C) Higher levels of PLEKHS1 expression in ATC tumors than PTCs. (D) PLEKHS1 mRNA and protein expression levels in 20 PTC tumors as determined using qPCR and immunoblotting, respectively. The quantification results (band intensity) in the bottom panel are shown in the top panel. (E) Positive correlation between PLEKHS1 mRNA and protein expression, as revealed by the analysis of combined results from both TC cell lines (A) and primary PTC tumors (D).

\subsection{Association Between PLEKHS1 Over-Expression and Metastases and Shorter Survival in PTCs}

Given the findings presented above, we sought to determine whether PLEKHS1 is associated with PTC progression and/or patient prognosis. Lymph node metastasis occurred in 50/93 of PTC patients and the primary tumors from these patients expressed significantly higher levels of PLEKHS1 mRNA than those without lymph node metastasis $(p=0.026)$ (Figure 3A and Table 1). Similarly, PLEKHS1 
expression was significantly higher in tumors from patients having distant metastases (12 cases) than those (81 cases) lacking metastases $(p=0.011)$ (Figure 3B and Table 1$)$. The relationship between PLEKHS1 expression and patient survival was further evaluated. The median expression level in tumors was used as a cut-off to divide the patients into low- and high-expressing groups. As shown in Figure 3C, the low-expressing group had significantly longer overall survival (OS) and disease-free survival (DFS) ( $p=0.011$ and 0.042 , respectively). When PLEKHS1 mRNA expression was used as a continuous variable, $p$ values for OS and DFS were $<0.001$ and 0.004 , respectively (Table 1). PLEKHS1 expression was not associated with patient age, gender, tumor size, or extrathyroidal extension (Table 1). We also analyzed the relationship between PLEKHS1 expression and clinico-pathological variables or TERT promoter statuses in 18 patients with ATC. As summarized in Table 2, there was no association of PLEKHS1 expression with age, gender, tumor size, survival, and TERT promoter mutations or TERT expression.

Table 1. Clinical characteristics and statistical comparison for the 93 PTC cases.

\begin{tabular}{|c|c|c|c|}
\hline Parameters $n=$ (No of Informative Cases) & Observations & $\begin{array}{c}\text { PLEKHS1 mRNA } \\
\text { Median (Min-Max) }\end{array}$ & $p$-Value \\
\hline Age at diagnosis $(n=93)$ & & & 0.365 \\
\hline Median (Min-Max) years & $51(15-97)$ & & \\
\hline$<55$ & $n=55$ & $1.43(0.21-139.30)$ & \\
\hline$\geq 55$ & $n=38$ & $1.67(0.37-811.88)$ & \\
\hline Gender $(n=93)$ & & & 0.980 \\
\hline Female & $n=67$ & $1.43(0.21-811.88)$ & \\
\hline Male & $n=26$ & $1.77(0.21-421.76)$ & \\
\hline Tumor size $(n=88)$ & & & 0.244 \\
\hline$\leq 2 \mathrm{~cm}(n=40)$ & $n=40$ & $1.06(0.21-11.30)$ & \\
\hline $2-4 \mathrm{~cm}(n=29)$ & $n=29$ & $2.09(0.21-811.88)$ & \\
\hline$>4 \mathrm{~cm}(n=19)$ & $n=19$ & $1.67(0.45-139.30)$ & \\
\hline Lymph node metastases $(n=93)$ & & & 0.026 \\
\hline No & $n=43$ & $1.17(0.21-421.76)$ & \\
\hline Yes & $n=50$ & $1.70(0.21-811.88)$ & \\
\hline Distant metastases $(n=93)$ & & & 0.011 \\
\hline No & $n=81$ & $1.28(0.21-421.76)$ & \\
\hline Yes & $n=12$ & $3.43(0.54-811.88)$ & \\
\hline Extrathyroidal extension $(n=81)$ & & & 0.833 \\
\hline No & $n=47$ & $1.26(0.21-97.07)$ & \\
\hline Yes & $n=34$ & $1.36(0.22-811.88)$ & \\
\hline TERT hotspot promoter mutation $(n=93)$ & & & 0.281 \\
\hline Wild-type & $n=64$ & $1.35(0.21-421.76)$ & \\
\hline Mutation C228T/C250T & $n=24 / 5$ & $1.86(0.37-811.88)$ & \\
\hline TERT mRNA expression $(n=93)$ & & & 0.603 \\
\hline No TERT mRNA & $n=40$ & $1.46(0.21-139.30)$ & \\
\hline TERT mRNA expressed & $n=53$ & $1.59(0.37-811.88)$ & \\
\hline Overall survival $(n=93)$ & & & $<0.001$ \\
\hline Alive & $n=32$ & $1.17(0.21-139.30)$ & $95 \% \mathrm{CI}=1.002-1.007$ \\
\hline Dead & $n=61$ & $2.17(0.37-811.88)$ & $H R=1.005$ \\
\hline Follow-up:median (min-max) years & $14.8(0.2-26.5)$ & & \\
\hline Disease-free survival $(n=93)$ & & & 0.004 \\
\hline No evidence of disease & $n=67$ & $1.26(0.21-421.76)$ & $95 \% \mathrm{CI}=1.001-1.007$ \\
\hline Relapsed/progression & $n=26$ & $2.09(0.34-811.88)$ & $H R=1.004$ \\
\hline Follow-up: Median (Min-Max) years & $13.5(0.1-26.5)$ & & \\
\hline
\end{tabular}

HR: hazard ratio, 95\% CI: 95\% confidence interval. Mann-Whitney U-test or Kruskal-Wallis Test was used for comparison between groups. Univariate Cox-regression was used for survival analysis, PLEKHS1 mRNA as. a continuous variable. $n$ : number. 
Table 2. Clinical characteristics and statistical comparison for the 18 ATC cases.

\begin{tabular}{|c|c|c|c|}
\hline Parameters (Number of Informative Cases) & Observations & $\begin{array}{c}\text { PLEKHS1 mRNA } \\
\text { Median (Min-Max) }\end{array}$ & $p$-Value \\
\hline \multicolumn{4}{|l|}{ Age at diagnosis $(n=18)$} \\
\hline Median (Min-Max) years & $77.5(54-91)$ & & 0.101 \\
\hline$<55$ years & $n=1$ & $1.03(1.03-1.03)$ & \\
\hline$\geq 55$ years & $n=17$ & $4.00(1.80-85.57)$ & \\
\hline Gender $(n=18)$ & & & 0.214 \\
\hline Female & $n=10$ & $4.68(2.92-85.57)$ & \\
\hline Male & $n=8$ & $2.76(1.03-18.91)$ & \\
\hline Tumor size $(n=18)$ & & & 0.251 \\
\hline$\leq 2 \mathrm{~cm}$ & $n=1$ & $1.03(1.03-1.03)$ & \\
\hline $2-4 \mathrm{~cm}$ & $n=2$ & $44.04(2.52-85.57)$ & \\
\hline$>4 \mathrm{~cm}$ & $n=15$ & $4.00(1.80-18.91)$ & \\
\hline TERT promoter mutation $(n=18)$ & & & 0.722 \\
\hline Wild-type & $n=8$ & $4.26(1.03-85.57)$ & \\
\hline Mutation (C228T/C250T) & $n=7 / 3$ & $3.67(1.80-18.91)$ & \\
\hline TERT mRNA expression $(n=18)$ & & & 0.888 \\
\hline No TERT mRNA & $n=2$ & $10.02(2.33-17.71)$ & \\
\hline TERT mRNA expressed & $n=16$ & $3.67(1.03-85.57)$ & \\
\hline Survival $(n=17)$ & & & 0.54 \\
\hline Alive & $n=1$ & $7.91(7.91-7.91)$ & \\
\hline Dead & $n=16$ & $3.25(1.03-85.57)$ & \\
\hline Follow-up: Median (Min-Max) months & $3(0-190)$ & & \\
\hline
\end{tabular}

$n$ : numbers. HR: hazard ratio, 95\% CI: 95\% confidence interval. Mann-Whitney U-test or Kruskal-Wallis Test was used for comparison between groups.

It is well known that TERT promoter mutations are associated with shorter patient survival in PTCs $[9,11]$, however, the majority of these patients carry a wt promoter and it is thus clinically important to discriminate between high and low risk patients in this category. To test whether PLEKHS1 can serve as such a marker, we determined its association with TERT hotspot promoter mutations and patient survival. As demonstrated previously, the presence of TERT promoter mutations predicted substantially shorter OS and DFS in PTC patients (Figure 3D). A comparison of PLEKHS1 expression between tumors that were wt and mutant for TERT hotspot promoter mutations did not show a difference, and moreover, PLEKHS1 expression was not associated with survival in cases with TERT promoter mutation ( $p=0.734$ and 0.621 for OS and DFS, respectively). However, in the group with a wt TERT promoter, higher PLEKHS1 expression was significantly associated with shorter OS ( $p=0.035)$ and showed a tendency toward shorter DFS, although not reaching statistical significance $(p=0.128)$ (Figure 3E).

In multivariate analyses, PLEKHS1 over-expression, age ( $\geq 55$ years old) and larger tumor size $(>4 \mathrm{~cm})$, but not gender and TERT promoter mutations, were independently associated with shorter patient OS (Table S1). However, PLEKHS1 over-expression and TERT promoter mutations had no effects on DFS, whereas tumor size remained as a predictor for poor DFS (Table S2).

We also analyzed the TCGA cohort of 393 patients with PTC for the relationship between PLEKHS1 expression and clinico-pathological variables. There was a significant association between PLEKHS1 over-expression and lymph node metastasis $(p=0.002)$ (Table S3), but not distant metastasis, likely due to the very few patients with distant metastasis (4/249) in the TCGA cohort. PLEKHS1 expression levels were not associated with patient survival (Table S3), whereas the presence of TERT promoter mutations predicted significantly shorter OS and DFS, as shown previously [9]. In PTCs with a wt 
TERT promoter, PLEKHS1 expression had no effects on patient survival, likely due to the low number of deaths during the follow-up period (7/281).
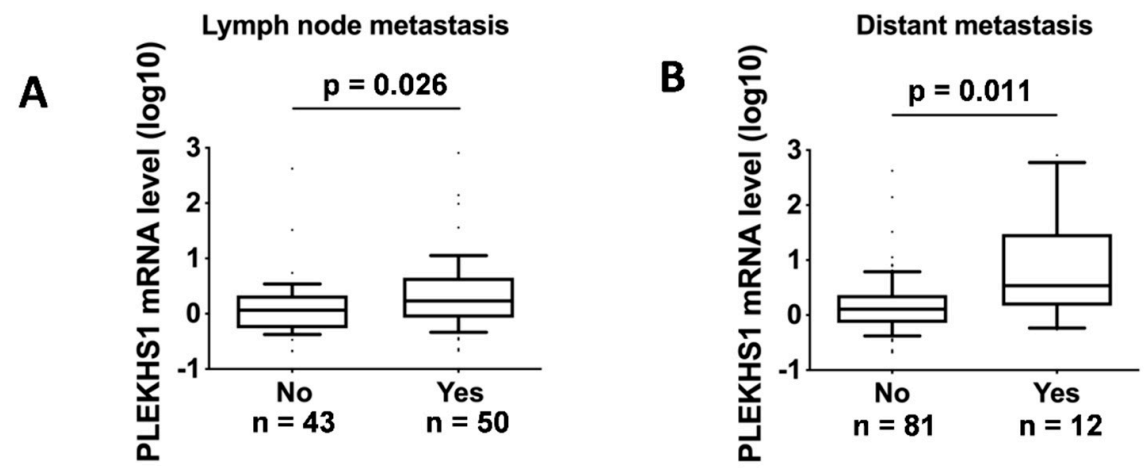

C
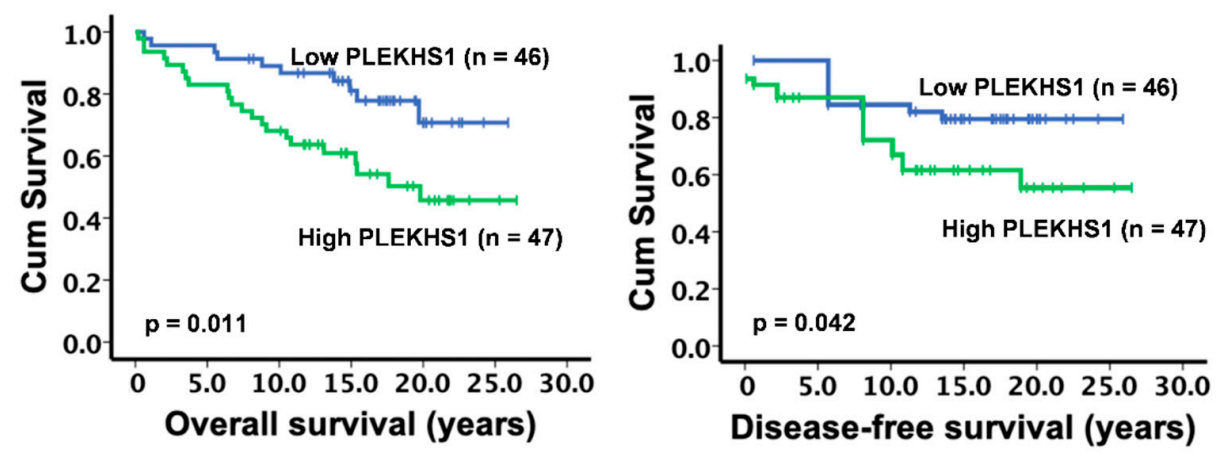

D
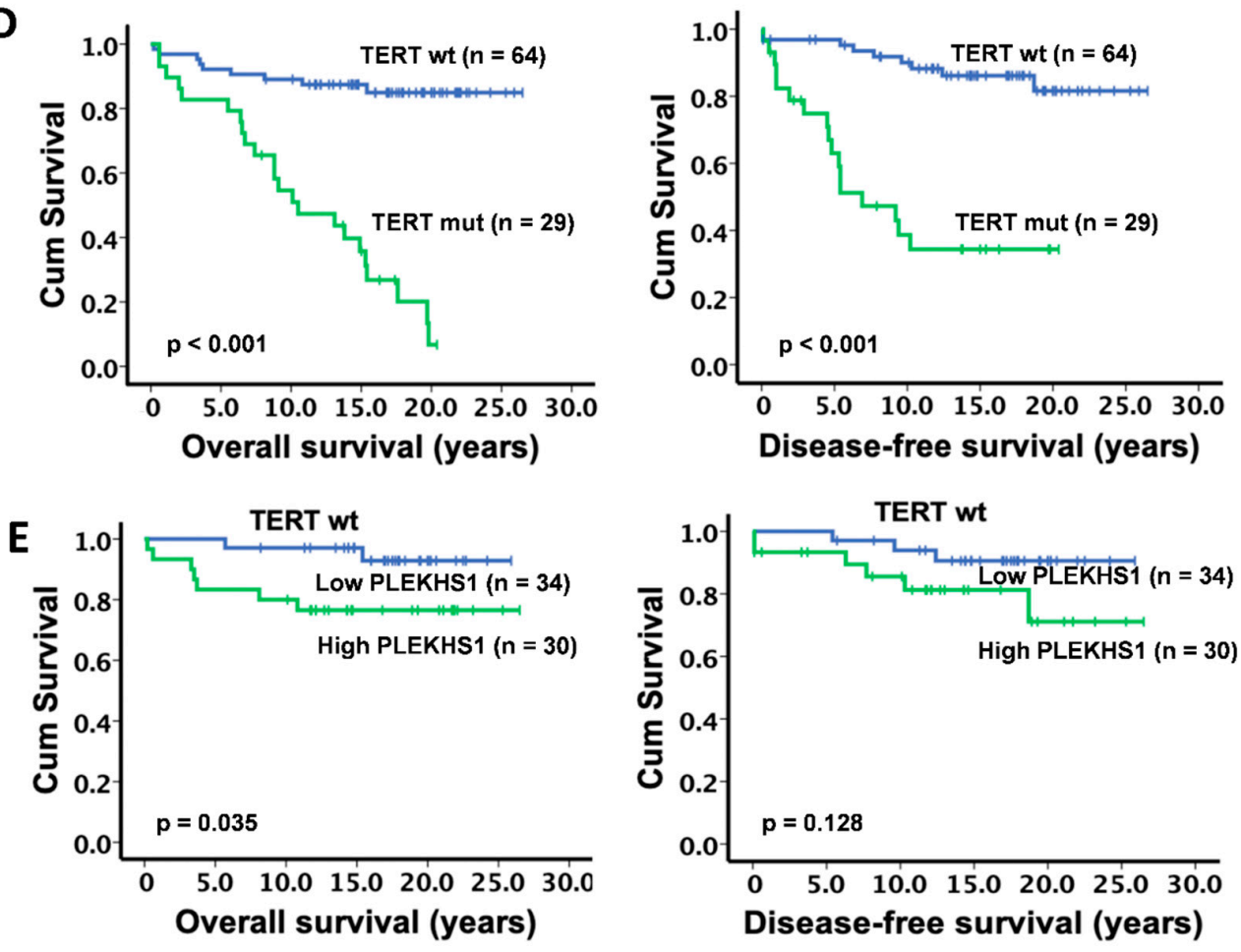

Figure 3. Higher PLEKHS1 expression is associated with metastasis and shorter survival in PTC patients. (A,B) Higher PLEKHS1 expression is associated with both lymph node and distant metastases. (C) Higher PLEKHS1 expression predicts significantly shortened overall and disease-free survival (OS \& DFS) time in PTC patients. (D) The presence of TERT promoter mutation is associated with significantly shorter OS and DFS in PTC patients. (E) The expression level of PLEKHS1 is associated with OS but not DFS in PTC patients with wt TERT promoter. 
2.4. Enhanced AKT Phosphorylation, Proliferation and Invasiveness Mediated by PLEKHS1 Over-Expression in TC Cells

Because pleckstrin homology domains (PH domains) contained in PLEKHS1 bind phosphoinositides, and PLEKHS1 interacts with PI3KR3 [19], we hypothesize that PLEKHS1 may be associated with the activation of the PI3K-AKT pathway. To explore this possibility, we over-expressed PLEKHS1 in TC cells (two PTC-derived and two ATC-derived cell lines) by ectopically introducing its expression vector, and determined the effect on AKT phosphorylation as an indicator of activation of the PI3K/AKT pathway. As shown in Figure 4A, the ectopic expression of PLEKHS1 substantially enhanced the level of phosphorylated AKT, but did not affect the total AKT expression significantly, in both PTC and ATC cells.

A

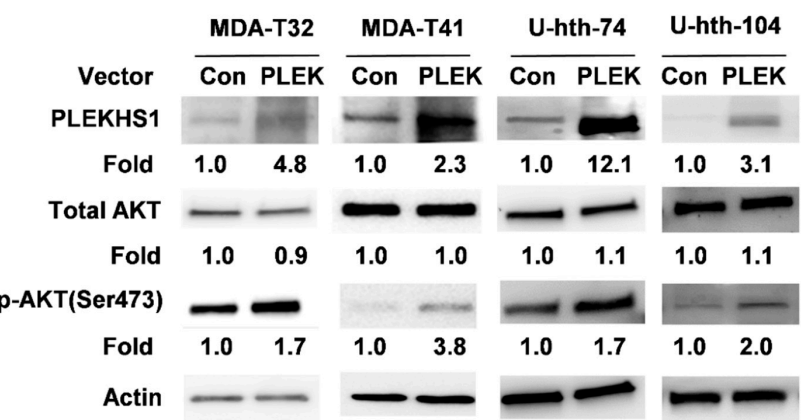

B
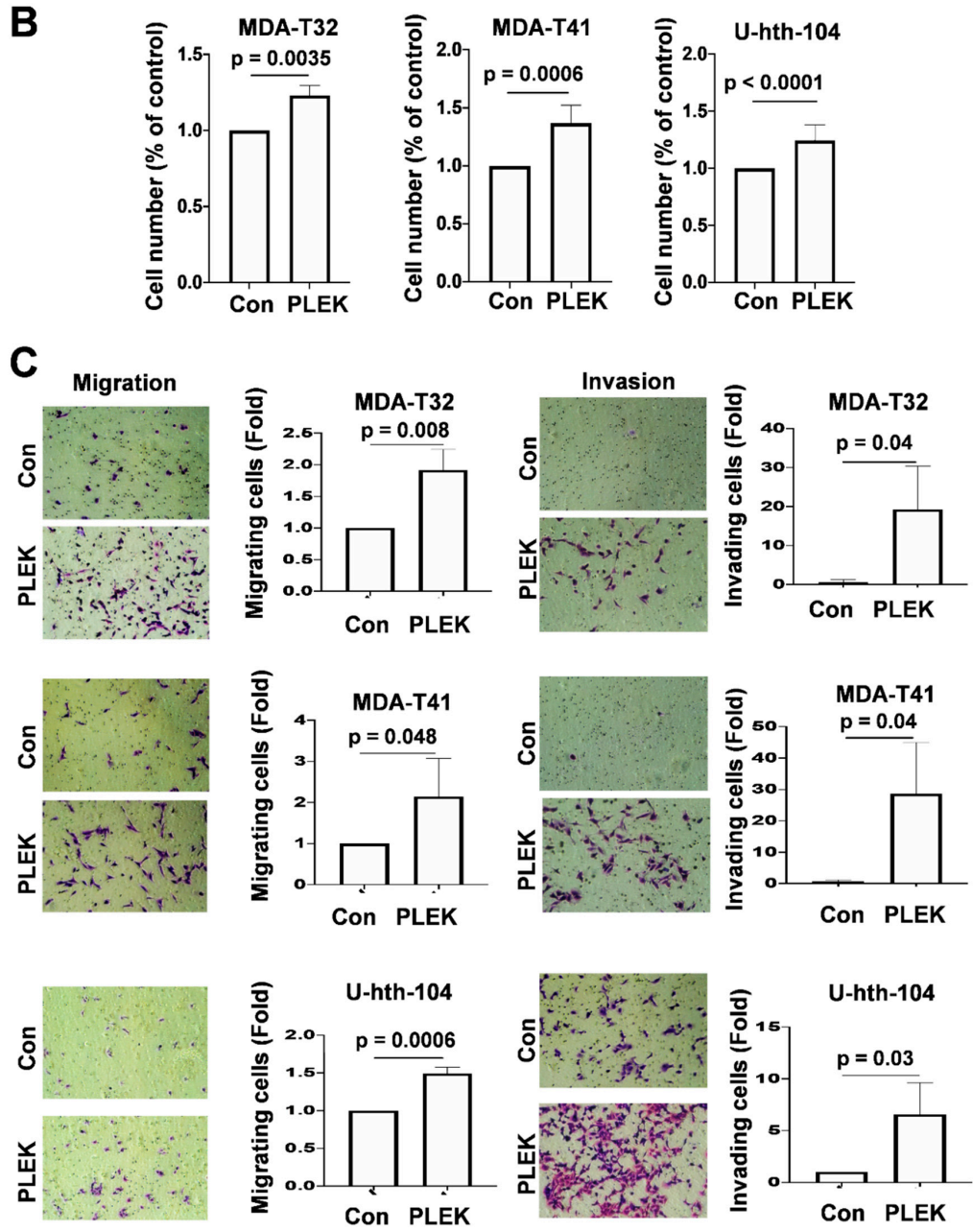
Figure 4. PLEKHS1 over-expression promotes AKT phosphorylation, proliferation and migration/invasion in PTC- and ATC-derived cells. (A) Increased AKT phosphorylation in PLEKHS1-transfected cells. MDA-T32, MDA-T41, U-hth-74, and U-hth-104 cells were transfected with control (Con) empty and PLEKHS1 (PLEK) expression vectors, respectively, and harvested $48 \mathrm{~h}$ post-transfection for total AKT and phosphorylated AKT levels using immunoblotting. One representative experiment out of three independent experiments is shown. The fold change for PLEKHS1, total and phosphorylated AKT is indicated (based on their signal intensity normalized to $\beta$-actin). (B) Increased cell proliferation of cells overexpressing PLEKHS1. MDA-T32, MDA-T41 and U-hth-104 cells above were incubated for $48 \mathrm{~h}$ and then counted. The number of control cells was set as $100 \%$. Shown are the results from three independent experiments. (C) Enhanced migration and invasion of PLEKHS1-overexpressed cells. MDA-T32, MDA-T41 and U-hth-104 cells above were analyzed for their migration and invasion capacities using a transwell assay system. Left: representative images of migration or invasion. Right: the quantification of migrating or invading cells based on three independent experiments.

The phosphorylated AKT exhibits an increased oncogenic activity, promoting cancer cell proliferation, survival and invasiveness or metastasis. Thus, we further determined alterations in proliferation and migration/invasion of PLEKHS1-overexpressed MDA-T32, MDA-T41 and U-hth-104 cells. As shown in Figure 4B, moderate, but significantly increased cell numbers were observed in PLEKHS1-transfected cells. Of note, these PLEKHS1-overexpressed cells acquired highly enhanced capacities in migration and invasion (Figure 4C). Especially for MDA-T32 and MDA-T41 cells, PLEKHS1 overexpression led to robust increases of invading cells (MDA-T32: 29-fold increase and MDA-T41: 43-fold increase) (Figure 4C). These results are highly consistent with PLEKHS1 overexpression in metastatic PTC patients.

\subsection{Association Between Demethylation and mRNA Expression of the PLEKHS1 Gene in PTCs}

Because the PLEKHS1 promoter mutation is rare in PTCs as described above, we sought to determine whether PLEKHS1 over-expression results from altered DNA methylation in tumors. To explore this, we first analyzed the methylation density at the PLEKHS1 locus in the TCGA database. The methylation data are available for 393 PTC tumors and 56 adjacent normal thyroid tissue samples. As shown in Figure 5A (Left panel), the overall methylation level was significantly lower in PTC tumors than their adjacent non-tumorous tissues $(p<0.0001)$. Moreover, a significantly inverse correlation between the methylation and mRNA expression of the PLEKHS1 gene was observed $(R=-0.184, p=0.0002)$ (Figure 5A, right panel). Furthermore, we specifically identified differentially methylated CpGs at the PLEKHS1 locus (Figure 5B), which include cg10627429 $(p<0.0001)$, cg11204562 $(-p<0.0001)$, and cg22618337 $(-p<0.0001)$ (Figure 5C). In all these three CpGs, the methylation level was inversely correlated with PLEKHS1 mRNA levels in PTC tumors $(R=-0.192,-0.268$ and -0.095 , respectively, $p<0.0001,<0.0001$ and $=0.06$, respectively) (Figure $5 C$ ), although the significance for cg22618337 is at a borderline level. Of note, all three CpGs are localized in the $5^{\prime}$ regulatory region of the PLEKHS1 gene (Figure 5B).

To see if this is the case in our PTC cohort, we further determined the methylation status of cg10627429 and cg11204562, two key CpGs associated with PLEKHS1 expression as described above. Pyrosequencing was performed on tumor DNA derived from 35 PTC patients and we observed differential methylation levels at cg10627429 and cg11204562 in these 35 tumors. Figure 6A shows the methylation of cg10627429 and cg11204562 in two patients, one with hypermethylation $(96 \%$ and $87 \%$ ) while the other with hypomethylation of two CpGs. The hypomethylated tumor expressed more than 1000-fold higher PLEKHS1 mRNA than did the hypermethylated one (422 vs. 0.41) (Figure 6B). Moreover, the analyses of 35 tumors reveal significantly inverse correlations between PLEKHS1 expression and methylation status of each CpG (cg10627429 and cg11204562, $r=-0.396$, and -0.428 , $p=0.019$ and 0.010 , respectively) or their combination $(r=-0.437, p=0.009)$ (Figure $6 \mathrm{C}$ ). 

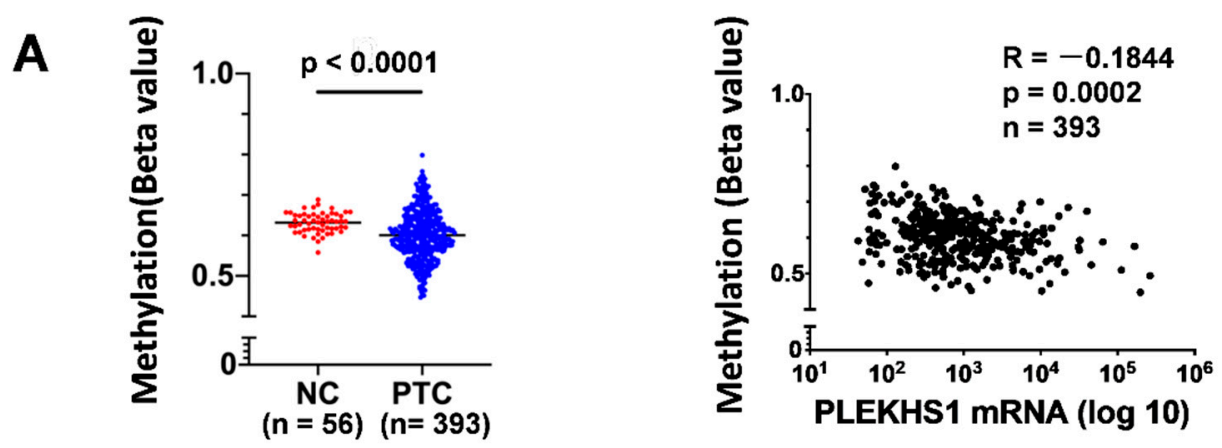

B

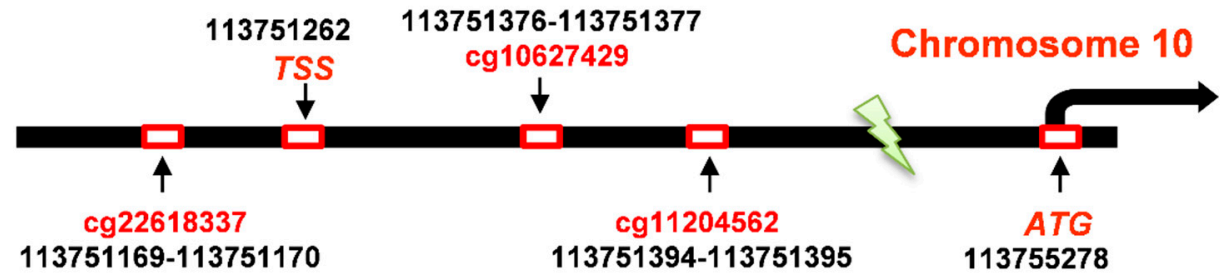

C
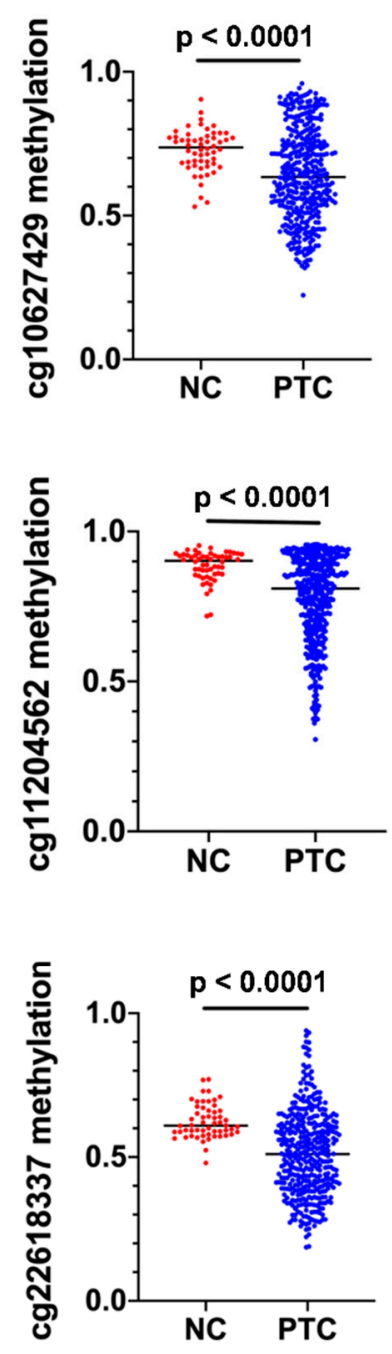
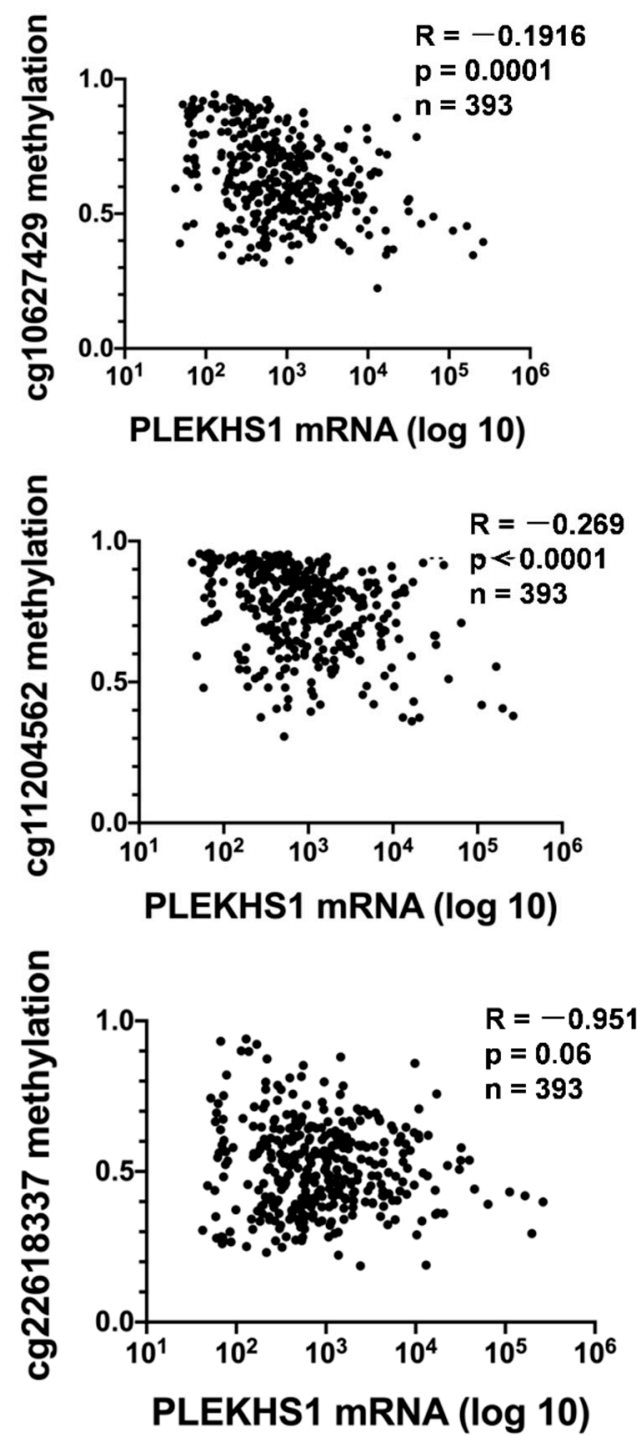
Figure 5. DNA methylation at the PLEKHS1 locus is inversely correlated with PLEKHS1 mRNA expression in the TCGA cohort of PTC patients. The analyses were performed on the DNA methylation at the PLEKHS1 locus from the TCGA cohort of PTC patients (393 tumors and 56 adjacent non-tumorous thyroid tissues) (downloaded from The Cancer Genome Atlas (TCGA) [20]. The level of DNA methylation was arbitrarily expressed as $\beta$ values (defined as the ratio of the signal intensity between methylated and unmethylated CpGs). PLEKHS1 mRNA levels were expressed as FPKM (Fragments Per Kilobase Million). (A) Left panel: Significantly reduced PLEKHS1 gene methylation in PTC tumors compared to their adjacent non-tumorous tissues. Right panel: Negative correlation between PLEKHS1 methylation and mRNA expression in PTC tumors. (B) The locations of 3 CpG sites with differential methylation at the PLEKHS1 locus between PTC tumors and non-tumorous tissues. TSS: Transcription start site. (C) The differential methylation levels at three specific CpG sites between PTC tumors and non-tumorous tissues and their negative correlation with PLEKHS1 mRNA expression in PTC tumors.

A

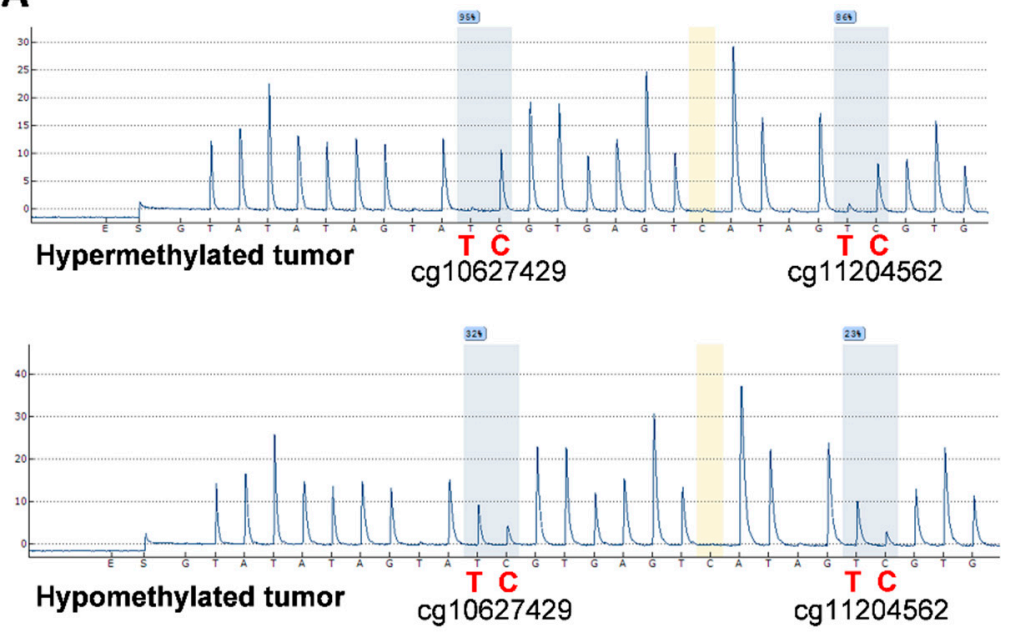

B

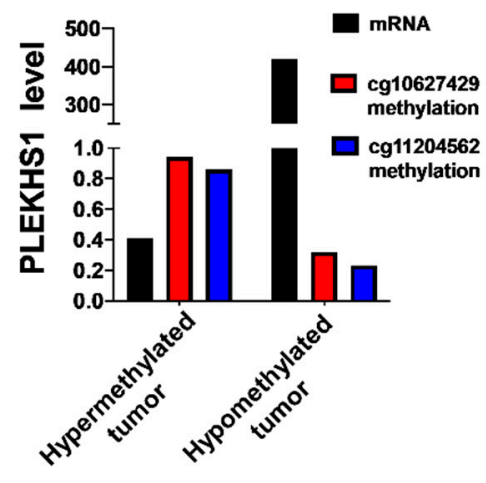

C
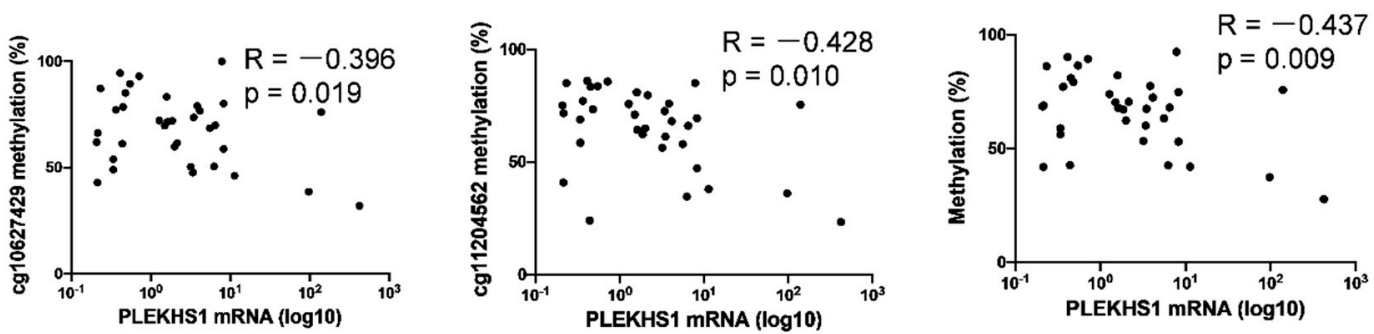

Figure 6. DNA methylation at the PLEKHS1 promoter is inversely correlated with PLEKHS1 mRNA expression in PTC patients. Pyrosequencing was performed to analyze the methylation status of cg10627429 and cg11204562 at the PLEKHS1 promoter in tumor DNA derived from 35 PTC patients, and the level of methylation at each CpG was expressed as percentage based on the PyroMark Q96 calculation. (A) The representative Pyrosequencing results from one patient with hypermethylation of both CpGs (Top) and one with hypomethylation (bottom). C (red): the methylated cytosine that is resistant to bisulfite conversion; $\mathrm{T}$ (red): the unmethylated cytosine that is conversed into uracil by bisulfite treatment. (B) PLEKHS1 mRNA levels and methylation quantification (based on Figure 6A) in these two tumors. (C) The inverse correlation between the methylation of cg10627429 and/or cg11204562 and PLEKHS1 expression in 35 PTC tumors.

\section{Discussion}

The PLEKHS1 gene encodes a PH domain-containing protein [17,21]_ENREF_14. It was previously shown that PLEKHS1 participated in blood glucose regulation and insulin resistance in obese rats, while its exact roles in physiological and pathological settings are currently unclear [21]. Nevertheless, 
the link of PLEKHS1 to human cancer has recently been established due to the identification of mutations in the PLEKHS1 promoter in several human malignancies including bladder, breast, lung, and thyroid cancer, as well as acute lymphocytic leukemia [17]. These mutations are single-nucleotide substitutions in the PLEKHS1 proximal promoter, among which two are hotspot mutated sites $50 \mathrm{bps}$ upstream from the first intron, predominately $\mathrm{C}>\mathrm{T}$ or $\mathrm{G}>\mathrm{A}$ transitions [17]. Approximately $40 \%$ of urothelial bladder tumors carry PLEKHS1 promoter mutations, exhibiting the highest frequency in all analyzed cancer types in TCGA datasets [17]. More interestingly, Weinhold et al. observed that the PLEKHS1 promoter mutation was present in 1/9 of thyroid tumors [17], with an occurrence rate largely similar to that of TERT promoter mutations in PTCs [16]. However, we screened 111 primary PTC and ATC tumors and found the PLEKHS1 promoter mutation in a single PTC sample. All five TC-derived cell lines harbor a wt promoter. Our results thus indicate that the PLEKHS1 promoter mutation is a rare genetic event in PTCs and ATCs.

The key finding in the present study is the association between PLEKHS1 expression and PTC outcomes. Higher levels of PLEKHS1 mRNA are associated with both lymph node and distant metastases and predict shorter OS and DFS in PTC patients. Moreover, PLEKHS1 expression still serves as a prognostic factor for poor outcomes in PTCs with a wt TERT promoter. Because the vast majority of PTCs lack TERT promoter mutations and reliable prognostic factors for these patients are urgently needed, our present observations might be clinically useful. As of this, future applications of our initial observations, such as PLEKHS1 immunohistochemical analyses in PTCs, could merit future attention as potential clinical discriminators of poor-prognosis cases when the TERT promoter mutational screening is negative.

Consistent with the present results, Pignot et al. recently observed that PLEKHS1 was more frequently over-expressed in muscle-invasive bladder cancer (MIBC), an aggressive subtype of the disease, and its over-expression independently predicted shorter progression-free survival [18]. Moreover, PLEKHS1 over-expression promoted non-muscle invasive bladder cancer (NMIBC) progression to aggressive MIBC [18]. It is thus evident from these observations that PLEKHS1 plays an oncogenic role in the pathogenesis of PTC and bladder cancer. Even so, little is known about the functional activity of PLEKHS1. Pleckstrin homology domains (PH domains) can bind phosphoinositides, and are known for different ligand binding sites and for specificity of different ligands, thereby triggering intracellular signaling. Because PLEKHS1 interacts with PI3KR3 [19], while the AKT signaling is required for the PTC pathogenesis [22], we sought to determine its effect on the PI3K-AKT signaling. Indeed, the ectopic expression of PLEKHS1 in thyroid cancer cells increased the abundance of phosphorylated AKT. We further show that PLEKHS1 overexpression promotes proliferation and invasiveness of both PTC- and ATC-derived cells. Especially, PTC cell lines overexpressing PLEKHS1 exhibited a dramatic increase in their invasive capacity. This observation suggests the involvement of PI3K-AKT in PLEKHS1-mediated PTC progression. Further studies are required to elucidate how exactly PLEKHS1 enhances AKT phosphorylation and thereby contributes to aggressive PTCs.

It is currently unclear how PLEKHS1 expression is regulated. Interestingly, PLEKHS1 mRNA abundance in the mutant PLEKHS1 promoter-bearing PTC tumor was the fourth highest among all PTCs. However, the PLEKHS1 promoter mutation occurs in almost the half of bladder tumors, but the presence of the mutation was not correlated with its mRNA expression [18], indicating a negligible effect of this genetic event on the PLEKHS1 transcription. However, Weinhold et al. showed that the mutant PLEKHS1 promoter-bearing tumors expressed lower levels of PLEKHS1 mRNA according to their results obtained from the TCGA datasets [17]. Given these inconsistent observations, further molecular and biochemical dissections are apparently required to ascertain how exactly this mutational event influences the PLEKHS1 gene transcription and contributes to oncogenesis. Based on the analysis of the TCGA cohort of PTC, we observed differential levels of DNA methylation at the PLEKHS1 locus between normal thyroid tissues and PTC tumors, and moreover, a significantly negative correlation between PLEKHS1 gene methylation and its mRNA expression was present in 
PTC tumors. Similarly, such an inverse correlation was observed in urothelial bladder tumors, too (Spearman correlation $\left.=-0.45, p=8.34 \times 10^{-22}\right)[23,24]$. Interestingly, all three CpGs are located in the PLEKHS1 promoter region. By determining the methylation status of these CpGs in the present cohort of PTC tumors, we further demonstrate an inverse association of the methylation with PLEKHS1 mRNA abundance. Thus, these results collectively suggest that the demethylation of the PLEKHS1 promoter induces an open chromatin locally, thereby contributing to PLEKHS1 over-expression during the pathogenesis of PTCs and bladder cancer. Further studies are required to elucidate the mechanisms underlying demethylation at the PLEKHS1 locus during oncogenesis.

In addition to the hotspot mutation in the PLEKHS1 promoter region as described above, its mutations in encoding sequences are also identified in bladder cancer and other malignancies $[17,23,25]$; and these mutations can be missense, truncating or inframe shifting. It remains unclear about the functional consequences of these different mutations, which calls for further investigations. Nevertheless, none of 399 PTC tumors were observed to bear mutations in the PLEKHS1 encoding region, as determined using exon sequencing analyses [5]. This finding, together with our present results, suggests that the aberrant DNA demethylation rather than its sequence alteration is targeted for the pathogenesis of thyroid carcinomas.

\section{Patients and Methods}

\subsection{Patients}

The study includes 93 patients with PTC and 18 patients with ATC, previously described and characterized by Yuan et al. [9]. Ethical permission was obtained from the Regional Ethics Committee (Dnr 2015/959-31 on 24-07-2015) in Stockholm, and informed consent was given prior to sample collection. The PTC and ATC diagnoses were made by histopathological examination according to the 2004 World Health Organization (WHO) classification [26]. We excluded the patients diagnosed as follicular variant of PTC to avoid potential inclusion of non-invasive follicular thyroid neoplasms with papillary-like nuclear features according to the more recent WHO classification from 2017 [1]. PTC patients were operated 1987-2005 and ATC patients 1989-2007. Tumor samples were collected post-operatively and kept frozen at $-80{ }^{\circ} \mathrm{C}$ until use following an established procedure where sample quality has been demonstrated over time [27]. After the exclusion of follicular variant of PTC, PTC samples were collected from the biobank and reviewed by a pathologist to confirm the representation of tumor cells in the frozen specimen. Clinical data, including age at diagnosis, gender, tumor size, lymph node, and distant metastasis status, was then retrospectively collected by reviewing the patient charts for the PTC cohort. Of the 12 patients with distant metastasis, two cases exhibited distant metastasis at diagnosis while the rest of the patients developed distant metastasis during the follow-up period. Follow-up data on OS (endpoints: dead or alive) and DFS (endpoints: relapsed/progression or disease-free) was also documented for PTC patients. Relapsed/progression was defined as local recurrence or metastasis during follow-up and disease-free was defined as no evidence of disease during the follow-up time after surgery. The last follow-up by reviewing patient charts was performed in September 2017. The detailed clinical information, selection process and frequency of TERT promoter mutations are reported and commented on as published [9]. Patient age, gender, lymph node status, and occurrence of distant metastases in relation to PLEKHS1 expression are summarized in Table 1 and 2 for PTC and ATC, respectively. Information on extrathyroidal extension (extension of tumor tissue into perithyroidal tissues including fat tissue according to AJCC 7th edition) was available for 81/93 PTC patients (Table 1) and TCGA cohort of PTCs.

The clinico-pathological data and TERT promoter status in the TCGA cohort of PTCs were downloaded via cBioPortal for Cancer Genomics [24,28,29], and PLEKHS1 mRNA abundances were arbitrarily expressed as RSEM (RNA-Seq by Expectation Maximization). The data for expression and methylation of the PLEKHS1 gene in PTC tumors and adjacent non-tumorous tissues were directly downloaded from The Cancer Genome Atlas (TCGA) [20] The levels of PLEKHS1 mRNA and DNA 
methylation were arbitrarily expressed as FPKM (Fragments Per Kilobase Million) and $\beta$ values (the

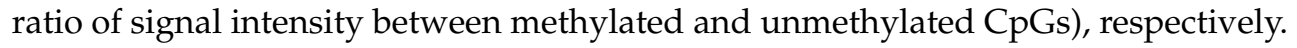

\subsection{Cell Lines and Cell Culture}

The study included five TC-derived cell lines (MDA-T32, MDA-T41, U-hth-74, U-hth-104, and SW1736). ATC-derived cell lines U-hth-74, U-hth-104 and SW1736 were obtained from Dr. N-E Heldin and cytogenetically characterized [30]. Short tandem repeats (STR) genotyping for ATC-derived cell lines U-hth-74, U-hth-104 and SW1736 was recently performed and matched to previously published genotypes [9], while the PTC-derived cell lines MDA-T32 and MDA-T41 were purchased from ATCC in 2018. Cells were cultured in RPMI-1640 medium (Thermo Fisher Scientific, Waltham, MA) supplemented with 10\% fetal bovine serum (Thermo Fisher Scientific, Waltham, MA), $100 \mathrm{U} / \mathrm{mL}$ penicillin, $100 \mathrm{\mu g} / \mathrm{mL}$ streptomycin, and $4 \mathrm{mM} \mathrm{L-glutamine.}$

\subsection{DNA Extraction and Sanger Sequencing}

Genomic DNA was extracted from PTC tissues and cell lines using DNeasy Blood \& Tissue Kit (Qiagen, Hilden, Germany) and the AllPrep Mini Kit (Qiagen) according to the manufacturer protocols, respectively. The mutational hotspots in TERT promoter region and the BRAF V600E mutational status was analyzed as described previously $[9,11]$. Sanger sequencing was applied for analyses of the PLEKHS1 promoter mutation and performed at the KIGene core facility, Stockholm, Sweden, using the following primers: 5'-GAA TCC TCG GGA CAA GGC ACT-3' (Forward) and 5'-GTC AGT CCT ATT TCC CTC TGA CT-3' (Reverse). PCR was run for 40 cycles with an annealing temperature at 60 ${ }^{\circ} \mathrm{C}$, which generated PCR products of $241 \mathrm{bp}$ (Figure 1A). Sequencing data were analyzed by visual inspection of chromatograms and manual examination.

\subsection{RNA Extraction, Reverse Transcription and qPCR}

Total RNA was extracted from cell lines and primary thyroid tumors using Trizol-Reagent (Thermo Fisher Scientific), and mirVana miRNA Isolation Kit (Invitrogen, Carlsbad, CA, USA), respectively. RNA was reversely transcribed using High Capacity cDNA Reverse Transcription Kit (Thermo Fisher Scientific). qPCR was performed in QuantStudio 7 Flex Real-Time PCR System using SYBR Green (Thermo Fisher Scientific). Relative expression levels of PLEKHS1 mRNA were calculated based on the $\triangle \mathrm{CT}$ values and normalized to human $\beta 2-M$ expression [9]. The following primers were used in this study for PLEKHS1: 5'-GGT CCA GAC CAG GTC TCT GGA-3' (Forward) and 5'-CCC CAT CCT GGG TCT CTG GA-3' (Reverse). PCR annealing temperature was $60^{\circ} \mathrm{C}$, and products were $144 \mathrm{bp}$ long fragments.

\subsection{Plasmid Transfection}

PLEKHS1 expression plasmids were purchased from AddGene (Watertown, MA, USA). TC cells grown in 6-well plates were transfected with empty control or PLEKHS1 expression vectors by using Lipofectamine3000 (Thermo Fisher Scientific) according to the protocol provided.

\subsection{Western Blot Analysis}

Total cellular proteins were extracted from cell lines and PTC tumors using RIPA Lysis Buffer (Thermo Fisher Scientific) with 1\% Phenylmethanesulfonyl fluoride (Sigma-Aldrich, Darmstadt, Germany) with or without Protease Inhibitor Cocktail (Sigma-Aldrich, Darmstadt, Germany). For the analysis of phosphorylated AKT, cells were washed once with sodium orthovanadate-containing buffers to inhibit phosphatases prior to RIPA buffer lysis. Thirty micrograms of proteins were separated in Mini-PROTEAN TGX Gels (Bio-Rad Laboratories, Hercules, CA) and transferred to PVDF membranes using Trans-Blot Turbo Transfer Pack (Bio-Rad). Membranes were blocked with 5\% non-fat milk diluted in TBST, and then incubated with primary antibodies and secondary antibodies before being 
imaged with Clarity Max Western ECL Substrate (Bio-Rad, 1705062) and ChemiDoc MP Imaging System (Bio-Rad). The following primary antibodies were used: anti-PLEKHS1 (Novus Biologicals, H00079949-M07) at dilution 1:500, anti-AKT (Cell Signaling Technology, Boston, MA, USA, 9272) and phosphorylated-AKT (Cell Signaling Technology, 4051) at dilution 1:1000, and anti- $\beta$-Actin (Santa Cruz, sc-47778, Dallas, TX, USA) at dilution 1:50,000. Secondary antibodies include Goat Anti-Mouse IgG (H + L)-HRP Conjugate (Bio-Rad, 170-6516) and Goat Anti-Rabbit IgG (H + L)-HRP Conjugate (Bio-Rad, 170-6515).

\subsection{Pyrosequencing for DNA Methylation Analyses}

Genomic DNA was bisulfited-converted using EpiTect Bisulfite Kits (Qiagen, Hilden, Germany) followed by PCR amplification using PyroMark PCR Kit (Qiagen) with PLEKHS1 promotor specific primers. The reverse primer was biotin-labeled to purify the PCR product by binding to streptavidin-coated Sepharose beads (GE Healthcare, UK). PCR fragments were bound to Sepharose beads followed by repeated washing and denaturation. Beads were then mixed with sequencing primer and annealed of sequencing primer to DNA. The plate was then transferred to a PyroMark Q96 (Qiagen) to perform sequencing according to the manufacturer's instructions. The primers for PCR and sequencing were: PLEKHS1 PCR primers: 5'-TTT TTA GGA AGA TAT TGG TTA AGA TAT GG-3' (forward) and 5'-Biotin-TCC TAC CAA ACT TTA AAC CAT AAT CAC AAT-3' (reverse); PLEKHS1 sequencing primer: $5^{\prime}$ - GGG ATT TTT TTT TAA TGG TAG T-3'.

\subsection{Transwell Assays for Cell Migration and Invasion}

A transwell assay system was used to determine the migration and invasion capacities of TC cells as described [9,31]. MDA-T32, MDA-T41 and U-hth-104 cells transfected with control or PLEKHS1 expression vectors $\left(1 \times 10^{5}\right)$ were seeded into the upper chamber. The low chamber contained RPMI-1640 medium with 20\% FBS. The migrated cells were stained with crystal violet, counted and photographed $24 \mathrm{~h}$ later. For invasion assay, $50 \mu \mathrm{L}$ matrigel (Corning Life Sciences, Flintshire, UK) was first loaded into the bottom of the upper chamber followed by the identical procedure as described above.

\subsection{Statistical Analyses}

All statistical analyses were performed using IBM SPSS Statistics version 24 (IBM, Armonk, NY, USA). Student's $t$-test or Mann-Whitney U-test was used for comparison of differences between groups. Spearman's or Pearson's correlation coefficient was applied to determine correlation coefficient $r$. Survival analyses were performed with log-rank test and univariate Cox regression. OS and DFS were visualized with Kaplan-Meier plots. PLEKHS1 mRNA levels were classified into two groups based on the median separation. Multivariate analysis was performed with a Cox regression model based on variables that were significant in univariate Cox regression. $p$-values below 0.05 were considered as statistically significant.

\section{Conclusions}

The results presented herein reveal that PLEKHS1 is over-expressed in thyroid carcinomas including PTCs and ATCs, and its higher expression is associated with lethal ATCs as well as metastatic PTCs and shorter patient survival. Mechanistically, unknown oncogenic events may drive the aberrant demethylation of the PLEKHS1 locus, thereby enhancing its expression. Moreover, PLEKHS1 may contribute to the hyperactivity of AKT, and consequently promotes aggressive PTCs. Our findings are thus of both biological and clinical importance.

Supplementary Materials: The following are available online at http://www.mdpi.com/2072-6694/12/8/2133/s1, Table S1: Univariate and multivariate Cox regression analyses of overall survival in 93 patients with papillary thyroid carcinoma, Table S2: Univariate and multivariate Cox regression analyses of disease-free survival in 93 patients with PTC, Table S3: Clinical characteristics and statistical comparison for the 393 PTC cases in TCGA. 
Author Contributions: Conceptualization, X.X., X.Y., C.L. and D.X.; Data curation, N.M., N.W., C.C.J. and K.S.; Formal analysis, X.X., N.M. and N.W.; Funding acquisition, C.C.J., C.L. and D.X.; Investigation, N.M., X.Y., N.W., C.C.J. and K.S.; Methodology, X.X. and N.M.; Project administration, X.Y., K.S. and D.X.; Resources, C.C.J. and C.L.; Supervision, X.Y. and C.L.; Validation, N.W. and K.S.; Visualization, K.S.; Writing and original draft, X.X., X.Y. and D.X.; Writing, review and editing, N.M., C.C.J., C.L. and D.X. All authors have read and agreed to the published version of the manuscript.

Funding: The study was supported by grants from Swedish Cancer Society, the Swedish Research Council, Cancer Society in Stockholm, The regional agreement on medical training and clinical research (ALF) between Stockholm County Council, and Karolinska Institutet. Xiaotian Yuan was supported by the China Postdoctoral Science Foundation Grant (2019M652404).

Acknowledgments: We thank Lisa Anfalk for excellent assistance in tumor tissue collection and N-E Heldin (Uppsala University, Sweden) for ATC cell lines.

Conflicts of Interest: The authors declare no conflict of interest.

\section{References}

1. Lloyd, R.V. WHO Classification of Tumours of Endocrine Organs. In WHO/IARC Classification of Tumours, 4th ed.; WHO: Geneva, Switzerland, 2017; Volume 10.

2. Kitahara, C.M.; Sosa, J.A. The changing incidence of thyroid cancer. Nat. Rev. Endocrinol. 2016, 12, $646-653$. [CrossRef]

3. Cabanillas, M.E.; McFadden, D.G.; Durante, C. Thyroid cancer. Lancet 2016, 388, 2783-2795. [CrossRef]

4. Simoes-Pereira, J.; Capitao, R.; Limbert, E.; Leite, V. Anaplastic Thyroid Cancer: Clinical Picture of the Last Two Decades at a Single Oncology Referral Centre and Novel Therapeutic Options. Cancers 2019, 11, 1188. [CrossRef]

5. Cancer Genome Atlas Research Network. Integrated genomic characterization of papillary thyroid carcinoma. Cell 2014, 159, 676-690.

6. Ravi, N.; Yang, M.; Gretarsson, S.; Jansson, C.; Mylona, N.; Sydow, S.R.; Woodward, E.L.; Ekblad, L.; Wennerberg, J.; Paulsson, K. Identification of Targetable Lesions in Anaplastic Thyroid Cancer by Genome Profiling. Cancers 2019, 11, 402. [CrossRef] [PubMed]

7. Jeon, S.; Kim, Y.; Jeong, Y.M.; Bae, J.S.; Jung, C.K. CCND1 Splice Variant as A Novel Diagnostic and Predictive Biomarker for Thyroid Cancer. Cancers 2018, 10, 437. [CrossRef]

8. Yuan, X.; Larsson, C.; Xu, D. Mechanisms underlying the activation of TERT transcription and telomerase activity in human cancer: Old actors and new players. Oncogene 2019, 38, 6172-6183. [CrossRef] [PubMed]

9. Yuan, X.; Mu, N.; Wang, N.; Straat, K.; Sofiadis, A.; Guo, Y.; Stenman, A.; Li, K.; Cheng, G.; Zhang, L.; et al. GABPA inhibits invasion/metastasis in papillary thyroid carcinoma by regulating DICER1 expression. Oncogene 2019, 38, 965-979. [CrossRef] [PubMed]

10. Yuan, X.; Dai, M.; Xu, D. TERT promoter mutations and GABP transcription factors in carcinogenesis: More foes than friends. Cancer Lett. 2020. [CrossRef]

11. Liu, T.; Wang, N.; Cao, J.; Sofiadis, A.; Dinets, A.; Zedenius, J.; Larsson, C.; Xu, D. The age- and shorter telomere-dependent TERT promoter mutation in follicular thyroid cell-derived carcinomas. Oncogene 2014, 33, 4978-4984. [CrossRef]

12. Xing, M.; Liu, R.; Liu, X.; Murugan, A.K.; Zhu, G.; Zeiger, M.A.; Pai, S.; Bishop, J. BRAF V600E and TERT promoter mutations cooperatively identify the most aggressive papillary thyroid cancer with highest recurrence. J Clin Oncol. 2014, 32, 2718-2726. [CrossRef] [PubMed]

13. Melo, M.; da Rocha, A.G.; Vinagre, J.; Batista, R.; Peixoto, J.; Tavares, C.; Celestino, R.; Almeida, A.; Salgado, C.; Eloy, C.; et al. TERT promoter mutations are a major indicator of poor outcome in differentiated thyroid carcinomas. J. Clin. Endocrinol. Metab. 2014, 99, E754-E765. [CrossRef] [PubMed]

14. Hysek, M.; Paulsson, J.O.; Jatta, K.; Shabo, I.; Stenman, A.; Hoog, A.; Larsson, C.; Zedenius, J.; Juhlin, C.C. Clinical Routine TERT Promoter Mutational Screening of Follicular Thyroid Tumors of Uncertain Malignant Potential (FT-UMPs): A Useful Predictor of Metastatic Disease. Cancers 2019, 11, 1443. [CrossRef] [PubMed]

15. Wang, N.; Liu, T.; Sofiadis, A.; Juhlin, C.C.; Zedenius, J.; Hoog, A.; Larsson, C.; Xu, D. TERT promoter mutation as an early genetic event activating telomerase in follicular thyroid adenoma (FTA) and atypical FTA. Cancer 2014, 120, 2965-2979. [CrossRef] 
16. Liu, T.; Yuan, X.; Xu, D. Cancer-Specific Telomerase Reverse Transcriptase (TERT) Promoter Mutations: Biological and Clinical Implications. Genes 2016, 7, 38. [CrossRef]

17. Weinhold, N.; Jacobsen, A.; Schultz, N.; Sander, C.; Lee, W. Genome-wide analysis of noncoding regulatory mutations in cancer. Nat Genet. 2014, 46, 1160-1165. [CrossRef]

18. Pignot, G.; Le Goux, C.; Vacher, S.; Schnitzler, A.; Radvanyi, F.; Allory, Y.; Lallemand, F.; Delongchamps, N.B.; Zerbib, M.; Terris, B.; et al. PLEKHS1: A new molecular marker predicting risk of progression of non-muscle-invasive bladder cancer. Oncol. Lett. 2019, 18, 3471-3480. [CrossRef]

19. GeneCards. Available online: https://www.genecards.org/cgi-bin/carddisp.pl?gene=PLEKHS1 (accessed on 5 January 2019).

20. The Cancer Genome Atlas (TCGA). Available online: http://cancergenome.nih.gov/ (accessed on 5 January 2019).

21. Kotoh, J.; Sasaki, D.; Matsumoto, K.; Maeda, A. Plekhs1 and Prdx3 are candidate genes responsible for mild hyperglycemia associated with obesity in a new animal model of F344-fa-nidd6 rat. J. Vet. Med. Sci. 2016, 78, 1683-1691. [CrossRef]

22. Tavares, C.; Eloy, C.; Melo, M.; Gaspar da Rocha, A.; Pestana, A.; Batista, R.; Bueno Ferreira, L.; Rios, E.; Sobrinho Simoes, M.; Soares, P. mTOR Pathway in Papillary Thyroid Carcinoma: Different Contributions of mTORC1 and mTORC2 Complexes for Tumor Behavior and SLC5A5 mRNA Expression. Int. J. Mol. Sci. 2018, 19, 1448. [CrossRef]

23. Cancer Genome Atlas Research Network. Comprehensive molecular characterization of urothelial bladder carcinoma. Nature 2014, 507, 315-322.

24. cBioPortal for Cancer Genomics. Available online: https://www.cbioportal.org (accessed on 10 November 2019).

25. Nik-Zainal, S.; Davies, H.; Staaf, J.; Ramakrishna, M.; Glodzik, D.; Zou, X.; Martincorena, I.; Alexandrov, L.B.; Martin, S.; Wedge, D.C.; et al. Landscape of somatic mutations in 560 breast cancer whole-genome sequences. Nature 2016, 534, 47-54. [CrossRef] [PubMed]

26. DeLellis R: Pathology and Genetics of Tumours of Endocrine Organs. In WHO Classification of Tumours, 3rd ed.; WHO: Geneva, Switzerland, 2004.

27. Andreasson, A.; Kiss, N.B.; Juhlin, C.C.; Hoog, A. Long-term storage of endocrine tissues at—80 degrees C does not adversely affect RNA quality or overall histomorphology. Biopreservation Biobanking 2013, 11, 366-370. [CrossRef] [PubMed]

28. Cerami, E.; Gao, J.; Dogrusoz, U.; Gross, B.E.; Sumer, S.O.; Aksoy, B.A.; Jacobsen, A.; Byrne, C.J.; Heuer, M.L.; Larsson, E.; et al. The cBio cancer genomics portal: An open platform for exploring multidimensional cancer genomics data. Cancer Discov. 2012, 2, 401-404. [CrossRef] [PubMed]

29. Gao, J.; Aksoy, B.A.; Dogrusoz, U.; Dresdner, G.; Gross, B.; Sumer, S.O.; Sun, Y.; Jacobsen, A.; Sinha, R.; Larsson, E.; et al. Integrative analysis of complex cancer genomics and clinical profiles using the cBioPortal. Sci. Signal. 2013, 6, pl1. [CrossRef]

30. Lee, J.J.; Foukakis, T.; Hashemi, J.; Grimelius, L.; Heldin, N.E.; Wallin, G.; Rudduck, C.; Lui, W.O.; Höög, A.; Larsson, C. Molecular cytogenetic profiles of novel and established human anaplastic thyroid carcinoma models. Thyroid 2007, 17, 289-301. [CrossRef]

31. Guo, Y.; Yuan, X.; Li, K.; Dai, M.; Zhang, L.; Wu, Y.; Sun, C.; Chen, Y.; Cheng, G.; Liu, C.; et al. GABPA is a master regulator of luminal identity and restrains the aggressive disease in bladder cancer. Cell Death Differ. 2020, 27, 1862-1877. [CrossRef]

(C) 2020 by the authors. Licensee MDPI, Basel, Switzerland. This article is an open access article distributed under the terms and conditions of the Creative Commons Attribution (CC BY) license (http://creativecommons.org/licenses/by/4.0/). 